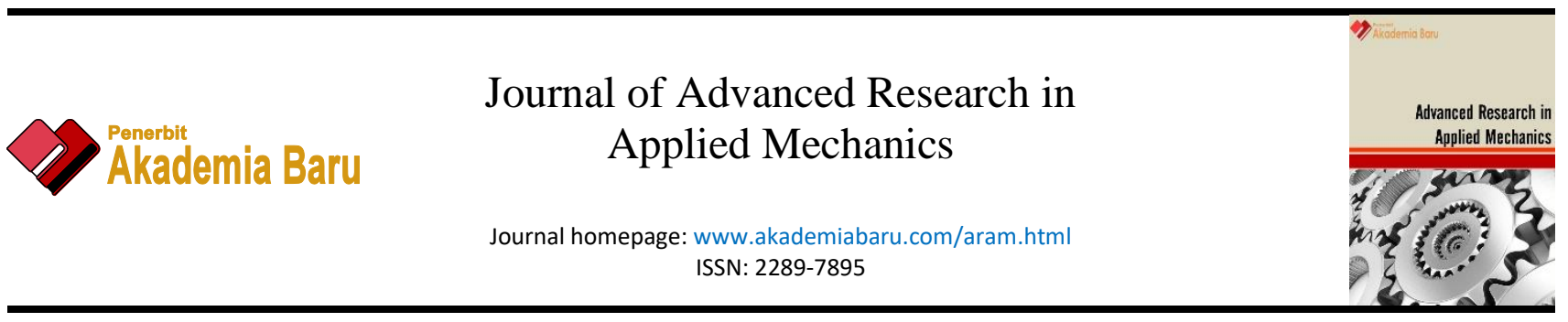

\title{
Richards' Equation: Transition Between Constitutive Equations and the Mechanics of Water Flow in Unsaturated Soil
}

\author{
Sunny Goh Eng Giap ${ }^{1,}$, , Noborio Kosuke ${ }^{2}$ \\ Faculty of Ocean Engineering Technology and Informatics, Universiti Malaysia Terengganu, 21030 Kuala Terengganu, Terengganu, Malaysia \\ Meiji University, 1-1-1 Higashimita, Tama-ku, Kawasaki 214-8571, Japan
}

\section{ABSTRACT}

Water flow in soil is normally governed by Richards' partial differential equation. Similarly, the equation can be used to investigate water infiltration in the soil. It is well understood that when water hits the surface of the topsoil, it infiltrates into the soil. However, the mechanics behind the infiltration of water is unknown to many researchers. This study aims to reveal the mechanics behind the well-known Richards' equation that has been used enormously in governing water flow in unsaturated soil since 1931. A classic case study of Haverkamp's water infiltration into Yolo Light Clay was used in the study. The Richards' equation has been discretized using finite difference method and the algebraic solution has been coded into Simply Fortran 2008. The partial differential equation of Richards is supported by two constitutive functions. The functions from Haverkamp and van Genuchten were compared. It is often for researcher to change between the hydraulic functions, which have limitation on the simulation outcome. The solution to overcome the limitation by changing the constitutive function variables was provided.

Keywords:

water flow in soil; unsaturated soil; water flux; water adsorption

Copyright @ 2020 PENERBIT AKADEMIA BARU - All rights reserved

\section{Introduction}

Richards' equation consists of a governing equation that is used to describe water flow in subsurface porous media such as soil [1]. It is continuously subjected to numerical investigation [29].The modeling of water distribution using the equation has important application in climate science, agriculture and also ecosystem management [10]. Among many applications, Zeide [11] has reported the use of Richards' equation in the tree growth modeling prediction. Soil scientist, agronomist, and irrigation engineer have found the equation useful to relate to plant water intake, and fertilizers and pesticides advection and dispersion in the soils [12].

The governing equation is represented by a partial differential equation. The equation is nonlinear that the soil water content $\left(\theta_{L}\right)$ has to be determined is influenced by its hydraulic conductivity $(K)$ and matric suction $\left(\psi_{m}\right)$, which both of them are depending on the soil water

\footnotetext{
* Corresponding author.

E-mail address: sunnygoh@gmail.com (Sunny Goh Eng Giap)
}

https://doi.org/10.37934/aram.73.1.1119 
content [13]. Adding to the complexity, Richards' equation governs the interface (unsaturated soil) is constantly subjected to the changing upper layer atmosphere and the changing lower layer of saturated soil that is governed Darcy's law. Richards' equation consists of two parts; the water flux expression that is similar to Darcy law, and the continuity equation is used to monitor the mass balance of water flux in and out of a control volume. The equation to describe the unsaturated flow can be written in three forms, as follows:

Head-based, $C\left(\psi_{m}\right) \frac{\partial \psi_{m}}{\partial t}=\frac{\partial}{\partial z}\left(K \frac{\partial \psi_{m}}{\partial z}\right)-\frac{\partial K}{\partial z}$

Mixed based, $\frac{\partial \theta_{L}}{\partial t}=\frac{\partial}{\partial z}\left(K \frac{\partial \psi_{m}}{\partial z}\right)-\frac{\partial K}{\partial z}$

Saturation-based, $\frac{\partial \theta_{L}}{\partial t}=\frac{\partial}{\partial z}\left(D\left(\theta_{L}\right) \frac{\partial \theta_{L}}{\partial z}\right)-\frac{\partial K}{\partial z}$

where detailed description on Eq. (1) was described in Rathfelder and Abriola [14]. Similarly, Kavetski et al., [15] and Zeng and Decker [16] have provided a detail explanation for mixed- and saturationbased of Richards' equation. To implement the equation, the soil water retention relation ( $\theta_{L}$ as a function of $\psi_{m}$ ) and the unsaturated hydraulic conductivity relation ( $K$ as a function of $\psi_{m}$ ) must be known from measurement [17].

Water infiltration is one of the common application for Richards' equation, and the water infiltrated medium is normally assumed homogeneous and isotropic [18]. In reality, the water flux regime could be affected depending on the locations in the soil that could be resulted from different transport coefficients such as the soil water retention curve and hydraulic conductivity relations, and also, a spatial variation of different saturated volumetric water contents [19]. Adding to this complexity, there are different constitutive functions to describe water retention curve and hydraulic conductivity relation, for example, van Genuchten [12], Campbell [21], and Haverkamp et al., [22]. The experimental dataset described by a constitutive function used in Richards' equation will be affecting the water infiltration profile.

The different water infiltration profiles resulted by different constitutive functions is a wellknown problem, but there is still no published record on the solution. This problem is common when one is applying published coefficients of a particular water retention curve equation, for example Haverkamp [22], to conduct simulation study, and then, attempt to change between constitutive function equations, for example, from Haverkamp et al., [22] to van Genuchten [20] equation. One would have recreated the dataset using Haverkamp equation coefficients first and then, redo the curve fitting on the dataset using Genuchten equation, hence, resulting different water infiltration profiles. Therefore, the first objective of the study is to provide a solution for the transition between constitutive equations and be able to produce similar water infiltration profile.

Richards' equation as described by Hopmans [17], it is a mass conservation equation and include the expression of Darcy law. An in-depth description on Richards' equation is also given by Germann [23] from the perspective of Navier-Stokes equation. However, take the case of water infiltration as an example, the influence of the coefficients $\left(D\left(\psi_{m}\right), K, \theta_{L}-\psi_{m}\right)$ as in Eq. (3) on water infiltration profile have not been discussed before. The second objective of this study is to reveal mechanics behind the Richards' equation as governed by the coefficients. 


\section{Methodology}

Richards' equation was reported as not behaving as mass conserving equation when using Eq. (1) [24]. Eqs. (2) and (3) were free from the mass conservation problem. The water-based Richards' equation as in Eq. (3) was chosen and used in this study because the volumetric water content appeared in the independent and dependent variables which would ease the programming effort. The $D\left(\psi_{m}\right)$ is the soil water diffusivity $\left(\mathrm{m}^{2} \cdot \mathrm{s}^{-1}\right)$ and it is given by,

$D\left(\psi_{m}\right)=K\left(\psi_{m}\right) \frac{\partial \psi_{m}}{\partial \theta_{L}}$

where $\frac{\partial \psi_{m}}{\partial \theta_{L}}$ is the derivative of matric suction $\left(\psi_{m},-\mathrm{m}\right)$ with respect to volumetric water content ( $\left.\theta_{L}, \mathrm{~m}^{3} \cdot \mathrm{m}^{-3}\right)$, and $K\left(\psi_{m}\right)$ is the hydraulic conductivity $\left(\mathrm{m} \cdot \mathrm{s}^{-1}\right)$ of the soil.

The constitutive functions from Haverkamp et al., [22] for the water retention curve $\left(\theta_{L}-\psi_{m}\right)$ and hydraulic conductivity $\left(K\left(\psi_{m}\right)\right)$ are given by,

$$
\begin{aligned}
& \psi_{m}=-10^{-2} \exp \left[\frac{\alpha\left(\theta_{s}-\theta_{r}\right)}{\theta_{L}-\theta_{r}}-\alpha\right]^{1 / \beta} \\
& K=K_{s} \frac{A}{A+\left(-100 \psi_{m}\right)^{B}}
\end{aligned}
$$

Van Genuchten [20] water retention curve and hydraulic functions are given below,

$$
\begin{aligned}
& \psi_{m}=-\frac{1}{\alpha_{1}}\left[\left(\frac{\theta_{s}-\theta_{r}}{\theta_{L}-\theta_{r}}\right)^{1 / m}-1\right]^{1 / n} \\
& K=K_{s}\left(\frac{\theta_{L}-\theta_{r}}{\theta_{s}-\theta_{r}}\right)^{L}\left\{1-\left[1-\left(\frac{\theta_{L}-\theta_{r}}{\theta_{s}-\theta_{r}}\right)^{1 / m}\right]^{m}\right\}^{2}
\end{aligned}
$$

where $\alpha, \alpha_{1}, A, B, \beta, L$ and $n$ are fitting parameters, $\theta_{s}$ is saturated water content $\left(\mathrm{m}^{3} \cdot \mathrm{m}^{-3}\right), \theta_{r}$ is residual water content $\left(\mathrm{m}^{3} \cdot \mathrm{m}^{-3}\right), K_{s}$ is saturated hydraulic conductivity $\left(\mathrm{m} \cdot \mathrm{s}^{-1}\right)$, and $m$ is also fitting parameter where $m=1-1 / n$.

To implement Richards' equation, Eq. (3) was discretized into algebraic equations using cellcentered finite-difference method, as follows, 


$$
\begin{aligned}
& \frac{\theta_{L(k)}{ }^{n+1}-\theta_{L(k)}{ }^{n}}{\Delta t}=\frac{K_{k+1 / 2}\left(\partial \psi_{m} / \partial \theta_{L}\right)_{k+1 / 2}}{\Delta z_{k}\left(0.5 \Delta z_{k+1}+0.5 \Delta z_{k}\right)}\left(\theta_{L(k+1)}{ }^{n+1}-\theta_{L(k)}{ }^{n+1}\right) \\
& -\frac{K_{k-1 / 2}\left(\partial \psi_{m} / \partial \theta_{L}\right)_{k-1 / 2}}{\Delta z_{k}\left(0.5 \Delta z_{k}+0.5 \Delta z_{k-1}\right)}\left(\theta_{L(k)}{ }^{n+1}-\theta_{L(k-1)}{ }^{n+1}\right) \\
& -\frac{K_{k+1 / 2} \vec{k}-K_{k-1 / 2} \vec{k}}{\Delta z_{k}}
\end{aligned}
$$

where $k$ indicates a center cell in z-direction of a cartesian coordinate system, $+1 / 2$ refers to the interface of cell located between cell $k$ and $k+1,-1 / 2$ refers to cell interface between cell $k-1$ and $k, n$ indicates the current time, $n+1$ indicates new time, and $\Delta$ refers to the difference of a parameter. Eq. (9) is oriented positive downward, so does Eq. (3). Complete description of the numerical scheme was explained in Goh and Noborio [25]. The equations were coded in Fortran.

Water infiltration phenomenon was used as a case study. The soil type used was Yolo light clay. A schematic diagram as in Figure 1(a) provides a simplistic view of the model environment.

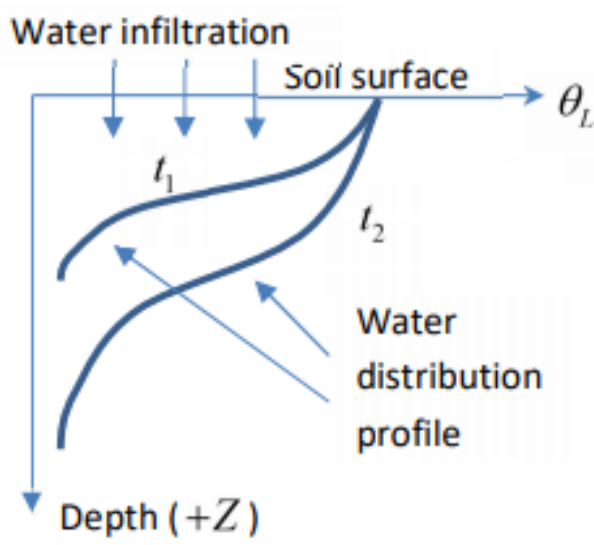

(a)

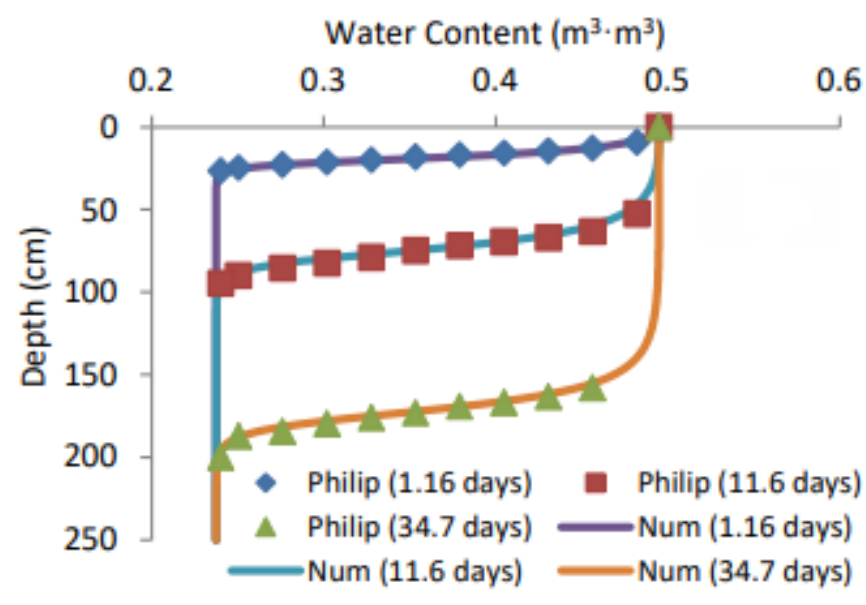

(b)

Fig. 1. Water distribution in the soil by: (a) a schematic diagram for soil water infiltration profile, and (b) water infiltration profile in Yolo light clay after 1.16, 11.6 and 34.7 days. Philip and Num referred to Philip's semianalytical solution and the Fortran coded numerical simulation, respectively

\section{Results}

\subsection{Validation of Simulation Results with Philip's Semianalytical Solution}

The Yolo light clay soil was initially wet with homogeneous soil moisture content of $0.2376 \mathrm{~m}^{3}$. $\mathrm{m}^{-3}$. The clay properties are shown in Table 1 . The water infiltration begins with the upper soil body saturated with $0.495 \mathrm{~m}^{3} \cdot \mathrm{m}^{-3}$ soil moisture content, and the lower soil body was assumed permeable to water, that is to say, water is free to move in and out. The total soil depth was taken as $2.5 \mathrm{~m}$. The soil depth was further divided into 100 cells, which each cell represents $2.5 \mathrm{~cm}$. Each cell was governed by Eq. (9) and the cells were simulated simultaneously in Fortran.

To make sure Richards' equation was programmed correctly, the simulation has to be validated either by experimentation or analytical solution dataset. In this case study, we used Philip's semianalytical solution [26-28] to validate the Fortran code. The results are shown in Figure 1(b). The 
R-squared showed a good match between Fortran simulation and the analytical solution of water infiltration profiles at $1.16,11.6$ and 34.7 days that were $0.9992,0.9988$ and 0.9994 , respectively.

\section{Table 1}

The soil type is Yolo light clay. The parameters used were retrieved from Haverkamp et al., [22]

\begin{tabular}{lllllll}
\hline Parameters & & & & & \\
$\alpha$ & $\theta_{r}\left(\mathrm{~m}^{3} \cdot \mathrm{m}^{-3}\right)$ & $\theta_{s}\left(\mathrm{~m}^{3} \cdot \mathrm{m}^{-3}\right)$ & $\beta$ & $A$ & $B$ & $K_{s}\left(\mathrm{~m} \cdot \mathrm{s}^{-1}\right)$ \\
\hline 739 & 0.124 & 0.495 & 4 & 124.6 & 1.77 & $1.23 \times 10^{-7}$ \\
\hline
\end{tabular}

Note: $\alpha, A, B$ and $\beta$ are fitting parameters, $\theta_{s}$ is saturated water content, $\theta_{r}$ is residual water content, and $K_{s}$ is saturated hydraulic conductivity.

\subsection{The problem of Transition Between Constitutive Equations}

Water retention curve is also known as water characteristic curve. There are many water characteristic curve equation [29], and new equation is continuously being proposed [30]. Hence, it would be impossible to address all the transition issues between all the equations. However, a simple case study would be discussed here as an example to demonstrate the technique used, which can be referred to as a transition method in solving similar problem.

Changing the characteristic curve equation, for example from Haverkamp to Genuchten equations, with the same dataset could result in different water infiltration profile, as showed in Figure 2(a). As shown in the Figure 2(a), at 34.7 days, van Genuchten equation simulation had under predicted the water infiltration profile. In achieving objective one that is to have an equal water infiltration profiles that transition from Haverkamp to van Genuchten equations, Eqs. (7) and (8) were modified to,

$$
\begin{aligned}
& \psi_{m}=-\frac{1}{\alpha_{1}}\left[\left(\frac{\theta_{s}-\theta_{r}}{\theta_{L}-\theta_{r}}\right)^{1 / m_{1}}-1\right]^{1 / n_{1}} \\
& K=K_{s}\left(\frac{\theta_{L}-\theta_{r}}{\theta_{s}-\theta_{r}}\right)^{L}\left\{1-\left[1-\left(\frac{\theta_{L}-\theta_{r}}{\theta_{s}-\theta_{r}}\right)^{1 / n_{2}}\right]^{m_{2}}\right\}^{2}
\end{aligned}
$$

where $m_{1}, n_{1}, m_{2}$, and $n_{2}$ are fitting parameters. The curve-fitting parameters were increased from 3 to 6 . The intention of the modification was to increase the ability of the Eqs. (10) and (11) to have a better curve-fitting ability than Eqs. (7) and (8). Figure 2(b) has proven the effectiveness of this method which was able to recreate the center of the water infiltration front, possible beyond 34.7 days.

Therefore, the best practice is to make the equation parameters unbound between the characteristic curve equation (Eq. (10)) and the hydraulic conductivity equation (Eq. (11)). This practice can be applied to other studies without much difficulty, however, most of the software, for example HYDRUS, does not allow such flexibility in the equation setting. 


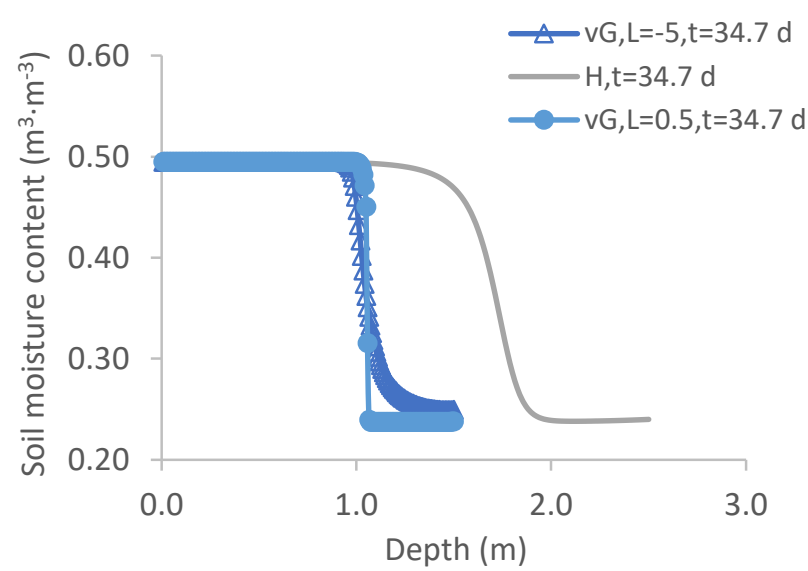

(a)

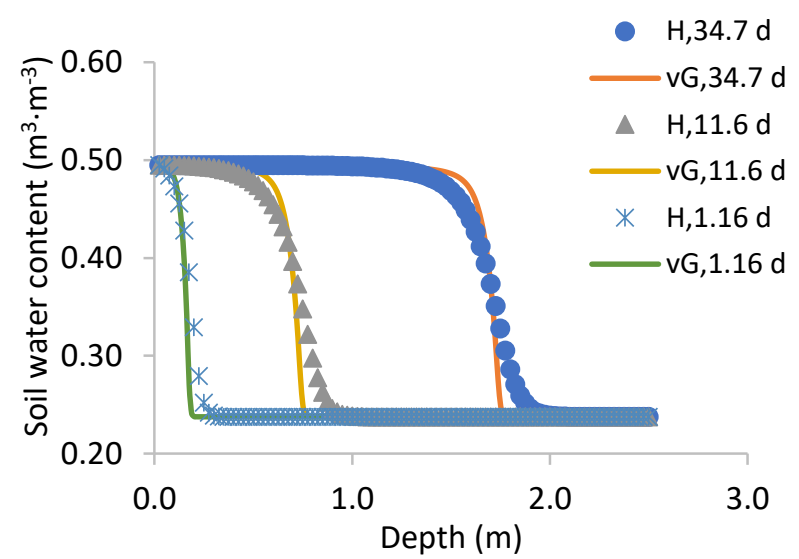

(b)

Fig. 2. Water infiltration profile for Genuchten and Haverkamp equations simulated at: (a) 34.7 days with $m, n, \alpha_{1}$ curve-fitted, while $L$ fixed at 0.5 and -5 ; and (b) 1.16, 11.6, 34.7 days with $L, m_{1}, n_{1}, m_{2}, n_{2}$, $\alpha_{1}$ curve-fitted. Note: vG and $\mathrm{H}$ corresponding to Genuchten and Haverkamp

\subsection{The mechanics of Richards' Equation}

Figure 2(b) showed that Richards' equation using improved Genuchten Eqs. (10) and (11) has significantly improved the simulation to match with those of the Haverkamp. The water infiltration front appeared sharply from 34.7 to 1.16 days of simulation. Although the improved Ganuchten is able to reproduce the waterfront center, Genuchten equation reproduces sharper waterfront than that of Haverkamp. The Genuchten waterfront appeared extrude in the high-water content region and intrude at the low water content region. This can be explained by the relation between hydraulic conductivity $(K)$ and soil moisture content, as in Figure 3(a), where the Genuchten curve is extruded in high water content region and intrude at the other region. Figure $3(\mathrm{~b})$ shows the rarely reported relation between $\frac{\partial \psi_{m}}{\partial \theta_{L}}$ and the soil moisture content. The graph shows a concave curve with a minimum was reached at $0.4586 \mathrm{~m}^{3} \cdot \mathrm{m}^{-3}$, which indicates a minimum gradient. Known that $D\left(\theta_{L}\right)$ in Eq. (4) consist of $K\left(\theta_{L}\right)$ and $\frac{\partial \psi_{m}}{\partial \theta_{L}}$, the former variable is always high at high soil moisture content as in Figure 3(a), whereas the latter variable would have to depend on the region soil wetness. This means that $\left(\partial \psi_{m} / \partial \theta_{L}\right)_{k-1 / 2}-\left(\partial \psi_{m} / \partial \theta_{L}\right)_{k+1 / 2}$ in space may not be always positive in value because it depends on the level of soil wetness. Whereas the $D\left(\theta_{L}\right)_{k-1 / 2}-D\left(\theta_{L}\right)_{k+1 / 2}$ is always positive for water infiltration front as long as the water is continuously infiltrating into the soil, which explain the increasing soil water content with time in the soil depth. 


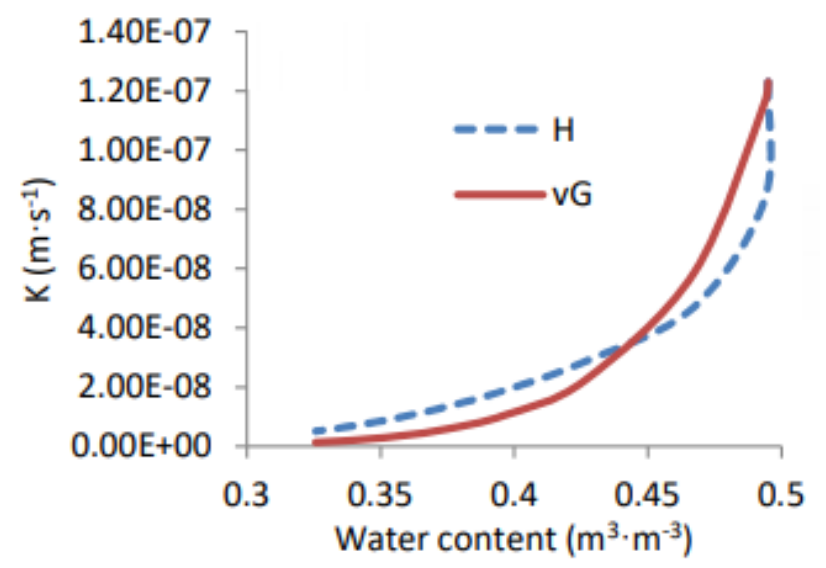

(a)

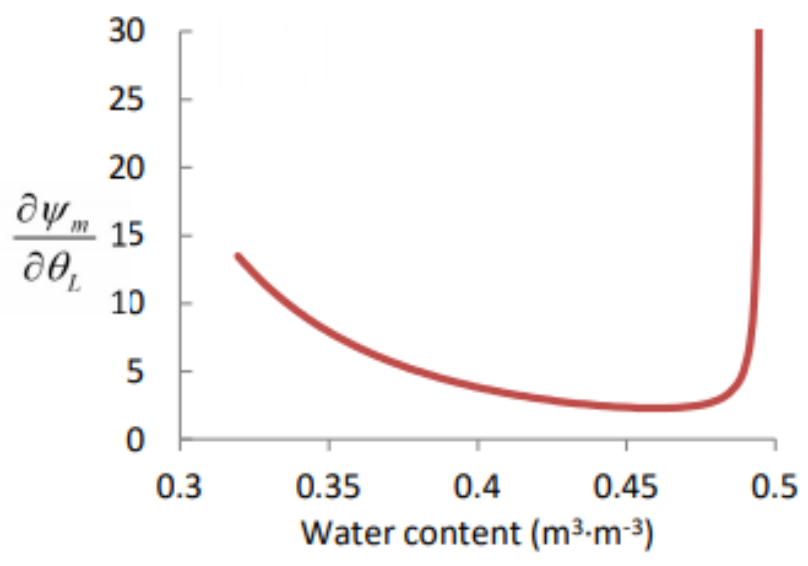

(b)

Fig. 3. (a) Hydraulic conductivity varies with soil moisture content, and (b) the derivative of soil matric suction with respect to moisture content varies with soil moisture content

\section{Conclusions}

Richards' equation is important to govern water flow in the unsaturated soil, which acts as an interface between the atmosphere and the groundwater. Richards' equation which is frequently used in conjunction with other constitutive equations (water characteristic curve and hydraulic functions) does not allow easy transition between equations, for example from Haverkamp to Genuchten. In the first objective, we have demonstrated that an easy modification of the existing Genuchten variables was able to reproduce water infiltration front. In the second objective, we have discussed the importance of understanding the variable in the Richards' equation in order to be able to understand the mechanics of water transport and also be able to describe the observed sharp water infiltration front produced from using Genuchten equation. The hidden property of the derivative of soil matric suction with respect to the soil moisture content is often overlooked has influenced on the influx of water into the soil.

\section{Acknowledgement}

I would like to acknowledge the financial support from Universiti Malaysia Terengganu and Meiji University.

\section{References}

[1] Richards, Lorenzo Adolph. "Capillary conduction of liquids through porous mediums." Physics 1, no. 5 (1931): 318333. https://doi.org/10.1063/1.1745010

[2] Ngo-Cong, Duc, Nam Mai-Duy, Diogenes L. Antille, and Martinus Th van Genuchten. "A control volume scheme using compact integrated radial basis function stencils for solving the Richards equation." Journal of Hydrology 580 (2020): 124240. https://doi.org/10.1016/i.jhydrol.2019.124240

[3] Berardi, Marco, Fabio Difonzo, and Luciano Lopez. "A mixed MoL-TMoL for the numerical solution of the 2D Richards' equation in layered soils." Computers \& Mathematics with Applications 79, no. 7 (2020): 1990-2001. https://doi.org/10.1016/i.camwa.2019.07.026

[4] Fengnan, Liu, Yasuhide Fukumoto, and Xiaopeng Zhao. "A linearized finite difference scheme for the Richards equation under variable-flux boundary conditions." Journal of Scientific Computing 83, no. 1 (2020): 16. https://doi.org/10.1007/s10915-020-01196-y

[5] Batista, Juan, Xiaozhe Hu, and Ludmil T. Zikatanov. "Auxiliary space preconditioning for mixed finite element discretizations of Richards' equation." Computers \& Mathematics with Applications 80, no. 2 (2020): 405-416. https://doi.org/10.1016/i.camwa.2019.09.011 
[6] De Rango, Alessio, Luca Furnari, Andrea Giordano, Alfonso Senatore, Donato D'Ambrosio, William Spataro, Salvatore Straface, and Giuseppe Mendicino. "OpenCAL system extension and application to the three-dimensional Richards equation for unsaturated flow." Computers \& Mathematics with Applications (2020).

https://doi.org/10.1016/j.camwa.2020.05.017

[7] Armiti-Juber, Alaa, and Christian Rohde. "On the well-posedness of a nonlinear fourth-order extension of Richards' equation." Journal of Mathematical Analysis and Applications (2020): 124005. https://doi.org/10.1016/i.jmaa.2020.124005

[8] Bassetto, Sabrina, Clément Cancès, Guillaume Enchéry, and Quang Huy Tran. "Robust Newton solver based on variable switch for a finite volume discretization of Richards equation." In International Conference on Finite Volumes for Complex Applications, pp. 385-393. Springer, Cham, 2020.

https://doi.org/10.1007/978-3-030-43651-3 35

[9] Gąsiorowski, Dariusz, and Tomasz Kolerski. "Numerical Solution of the Two-Dimensional Richards Equation Using Alternate Splitting Methods for Dimensional Decomposition." Water 12, no. 6 (2020): 1780. https://doi.org/10.3390/w12061780

[10] Daly, Edorado, and Amilcare Porporato. "A review of soil moisture dynamics: from rainfall infiltration to ecosystem response." Environmental engineering science 22, no. 1 (2005): 9-24. https://doi.org/10.1089/ees.2005.22.9

[11] B. Zeide. Tree Growth. In Encyclopedia of Ecology, S. E. Jørgensen and B. D. Fath, Eds. Oxford: Academic Press, 2008, pp. 3588-3594. https://doi.org/10.1016/B978-008045405-4.00219-6

[12] Taigbenu, Akpofure E. "Unsaturated Flow (Richards Equation)." In The Green Element Method, pp. 217-230. Springer, Boston, MA, 1999. https://doi.org/10.1007/978-1-4757-6738-4 8

[13] Hillel, Daniel. Environmental soil physics: Fundamentals, applications, and environmental considerations. Elsevier, 1998.

[14] Rathfelder, Klaus, and Linda M. Abriola. "Mass conservative numerical solutions of the head-based Richards equation." Water Resources Research 30, no. 9 (1994): 2579-2586. https://doi.org/10.1029/94WR01302

[15] Kavetski, D., P. Binning, and S. W. Sloan. "Adaptive time stepping and error control in a mass conservative numerical solution of the mixed form of Richards equation." Advances in water resources 24, no. 6 (2001): 595-605. https://doi.org/10.1016/S0309-1708(00)00076-2

[16] Zeng, Xubin, and Mark Decker. "Improving the numerical solution of soil moisture-based Richards equation for land models with a deep or shallow water table." Journal of Hydrometeorology 10, no. 1 (2009): 308-319. https://doi.org/10.1175/2008JHM1011.1

[17] J. W. Hopmans. 2.05 - Infiltration and Unsaturated Zone. Treatise on Water Science, P. Wilderer, Ed. Oxford: Elsevier, 2011, pp. 103-114. https://doi.org/10.1016/B978-0-444-53199-5.00031-2

[18] T. P. A. Ferré and A. W. Warrick. INFILTRATION. Encyclopedia of Soils in the Environment, D. Hillel, Ed. Oxford: Elsevier, 2005, pp. 254-260. https://doi.org/10.1016/B0-12-348530-4/00382-9

[19] Sposito, G. "Physical Properties and Processes: Scaling." (2016). https://doi.org/10.1016/B978-0-12-409548-9.09696-2

[20] Van Genuchten, M. Th. "A closed-form equation for predicting the hydraulic conductivity of unsaturated soils." Soil science society of America journal 44, no. 5 (1980): 892-898. https://doi.org/10.2136/sssaj1980.03615995004400050002x

[21] Campbell, Gaylon S. "A simple method for determining unsaturated conductivity from moisture retention data." Soil science 117, no. 6 (1974): 311-314. https://doi.org/10.1097/00010694-197406000-00001

[22] Haverkamp, Roland, Michel Vauclin, Jaoudat Touma, P. J. Wierenga, and Georges Vachaud. "A comparison of numerical simulation models for one-dimensional infiltration." Soil Science Society of America Journal 41, no. 2 (1977): 285-294. https://doi.org/10.2136/sssaj1977.03615995004100020024x

[23] Germann, P. F. "Macropores and macropore flow, kinematic wave approach." (2005): 393-402. https://doi.org/10.1016/B0-12-348530-4/00491-4

[24] Celia, Michael A., Efthimios T. Bouloutas, and Rebecca L. Zarba. "A general mass-conservative numerical solution for the unsaturated flow equation." Water resources research 26, no. 7 (1990): 1483-1496. https://doi.org/10.1029/WR026i007p01483 
[25] Goh, Eng Giap, and Kosuke Noborio. "Sensitivity analysis and validation for numerical simulation of water infiltration into unsaturated soil." International scholarly research notices 2015 (2015). https://doi.org/10.1155/2015/824721

[26] Philip, J. R. "The theory of infiltration: 1. The infiltration equation and its solution." Soil science 83, no. 5 (1957): 345-358. https://doi.org/10.1097/00010694-195705000-00002

[27] Philip, J. R. "Numerical Solution of Equations of the Diffusion Type with Diffusivity Concentration? Dependent. II." Australian Journal of Physics 10, no. 1 (1957): 29-42. https://doi.org/10.1071/PH570029

[28] Philip, J. Re. "Theory of infiltration." In Advances in hydroscience, vol. 5, pp. 215-296. Elsevier, 1969. https://doi.org/10.1016/B978-1-4831-9936-8.50010-6

[29] Haghverdi, Amir, Mohsen Najarchi, Hasan Sabri Öztürk, and Wolfgang Durner. "Studying Unimodal, Bimodal, PDI and Bimodal-PDI Variants of Multiple Soil Water Retention Models: I. Direct Model Fit Using the Extended Evaporation and Dewpoint Methods." Water 12, no. 3 (2020): 900. https://doi.org/10.3390/w12030900

[30] Zhang, Yuwei, Zhanping Song, Xiaolin Weng, and Yongli Xie. "A new soil-water characteristic curve model for unsaturated loess based on wetting-induced pore deformation." Geofluids 2019 (2019). https://doi.org/10.1155/2019/1672418 